\title{
Variabilité des nutriments foliaires de Quercus suber L dans différentes situations écologiques dans le massif des Maures (Var, France), et relations avec la production de liège
}

\author{
J Orgeas, G Bonin \\ Laboratoire de biosystématique et écologie méditerranéenne, université de Provence/Ura 1152 , \\ FST Saint-Jérôme, case 421 bis, 13397 Marseille cedex 20, France
}

(Reçu le 15 novembre 1994 ; accepté le 26 juillet 1995)

\begin{abstract}
Résumé - Un suivi spatiotemporel des nutriments foliaires a été effectué sur le chêne-liège (Quercus suber $L$ ), dans le massif des Maures (Var, France) simultanément à l'étude de l'épaisseur des cernes du liège. Ceci a permis de mettre en relation la production de liège avec les teneurs en éléments minéraux dans différentes conditions écologiques. Les concentrations foliaires des ions évoluent en fonction de la saison, ainsi que de l'état de dégradation et de la xéricité des parcelles. L'azote varie proportionnellement à l'activité physiologique de la feuille, alors que le calcium et le potassium y sont stockés progressivement. L'antagonisme calcium-magnésium a été confirmé. Par ailleurs, les variations des nutriments dues au débroussaillement sont estompées dès la reprise printanière du métabolisme. La croissance du liège primaire ou mâle est linéaire quelles que soient les conditions climatiques et les perturbations. La production de liège secondaire ou femelle n'est pas linéaire dans le temps (il y a augmentation de la production avec le temps et l'âge de l'arbre), mais elle reste très supérieure à la production de liège mâle. Les plus fortes productions de liège femelle sont influencées favorablement par les fortes teneurs en potassium et en azote du feuillage, et par des milieux humides et peu perturbés. Le magnésium est lié aux faibles épaisseurs de liège. Le sodium et le calcium ne semblent pas influencer cette production de liège.
\end{abstract}

Quercus suber L / nutriments foliaires / production de liège / perturbation / conditions écologiques

Summary - Variability of foliar nutrient contents of Quercus suber $L$ in several ecological conditions in the Maures Mountains (Var, France), and relations with cork production. A monitoring of foliar nutrients has been undertaken on cork-oaks (Quercus suber $L$ ), in the Maures Mountains (Var, France). Simultaneously, the thickness of cork age rings has been studied to define the relationship of the cork production to nutrient contents under different ecological conditions. Foliar concentrations of ions evolved according to the season, the degradation state and the xericity of plots. 
Nitrogen appeared proportional to the physiological activity of the leaf, while calcium and potassium were stocked gradually. The calcium-magnesium antagonism was confirmed. Furthermore, changes of the nutrient concentrations in the undergrowth-clearing sites are reduced from the spring resumption of the metabolism. The growth of primary cork is linear with time, irrespective of year, climatic conditions and perturbations. The secondary (or reproductive) cork production is not linear with time and the age of trees, but it remains much higher than the primary cork production. The higher secondary cork productions are linked to the high contents of potassium and nitrogen, and to the moist and weakly disturbed plots. Magnesium is linked to small width thicknesses of cork age rings. Sodium and calcium do not appear to influence this cork production.

\section{Quercus suber L / foliar nutrients / cork production / disturbance / ecological conditions}

\section{INTRODUCTION}

Si le diagnostic foliaire a servi en de nombreuses circonstances en agronomie sur les arbres des régions médio-européennes, il a été peu utilisé à ce jour dans une étude liant les variations spatiales et temporelles des nutriments sur des végétaux sclérophylles méditerranéens. Les thèses d'EdDerfoufi (1986), Hasnaoui (1992), Ballini (1993) et Livrelli (1993) sont parmi les seuls travaux à aborder le problème des variations liées aux conditions écologiques et phénologiques sur des ligneux méditerranéens avec les travaux de Chapin (1980) sur les végétaux sclérophylles en général. Specht et Rundel (1990) ont plutôt travaillé sur la relation entre le degré de sclérophyllie et la teneur en quelques nutriments principaux. Dans le cas de l'évolution temporelle des nutriments, le problème est abordé ici pour le chêne-liège (Quercus suber $L$ ) qui occupe dans le sud-est français un territoire délimité aux massifs des Maures et de l'Estérel. Cette essence a été favorisée, dans ce secteur géographique, par la disparition du pin maritime dans un contexte souvent défavorable à l'exploitation du liège. Aussi les suberaies occupent-elles des biotopes très divers allant des matorrals secs thermophiles aux sylves de versant nord où s'infiltrent des feuillus. Dans ce large éventail de situations écologiques, il convient de discerner les milieux où le chêne-liège peut être considéré en situation optimale pour une exploitation rationnelle, et ceux où il végète dans un contexte de maquis arboré. Dans ce but, une étude des bilans nutritionnels au niveau des feuilles au cours de plusieurs saisons permettra de comparer les réactions écophysiologiques des peuplements dans les différentes situations écologiques et de les lier à la production de liège dans la perspective d'une étude finalisée pour une meilleure exploitation des suberaies des Maures. L'effet du débroussaillement sur les bilans nutritionnels sera aussi pris en compte afin d'apprécier l'impact d'une telle pratique sur les arbres en place.

\section{MATÉRIELS ET MÉTHODES}

Le choix des stations d'étude a fait l'objet d'un plan d'échantillonnage incluant en premier lieu une prospection exhaustive de la moitié occidentale du massif des Maures à l'aide de photographies aériennes infrarouge couleur et de cartes topographiques. Les placettes (82) ont été sélectionnées et comparées entre elles à l'aide de classifications ascendantes hiérarchiques (CAH) utilisant 20 paramètres stationnels comme variables. Dix de ces placettes ont été retenues (voir tableau I) comme étant les plus représentatives des différentes classes de l'arbre de classification ascendante hiérarchique (c'est-à-dire des différents types de situations écologiques rencontrées).

Pour mettre en évidence l'importance des facteurs environnementaux et pour clarifier la lecture des courbes d'évolution individuelle des ions, 


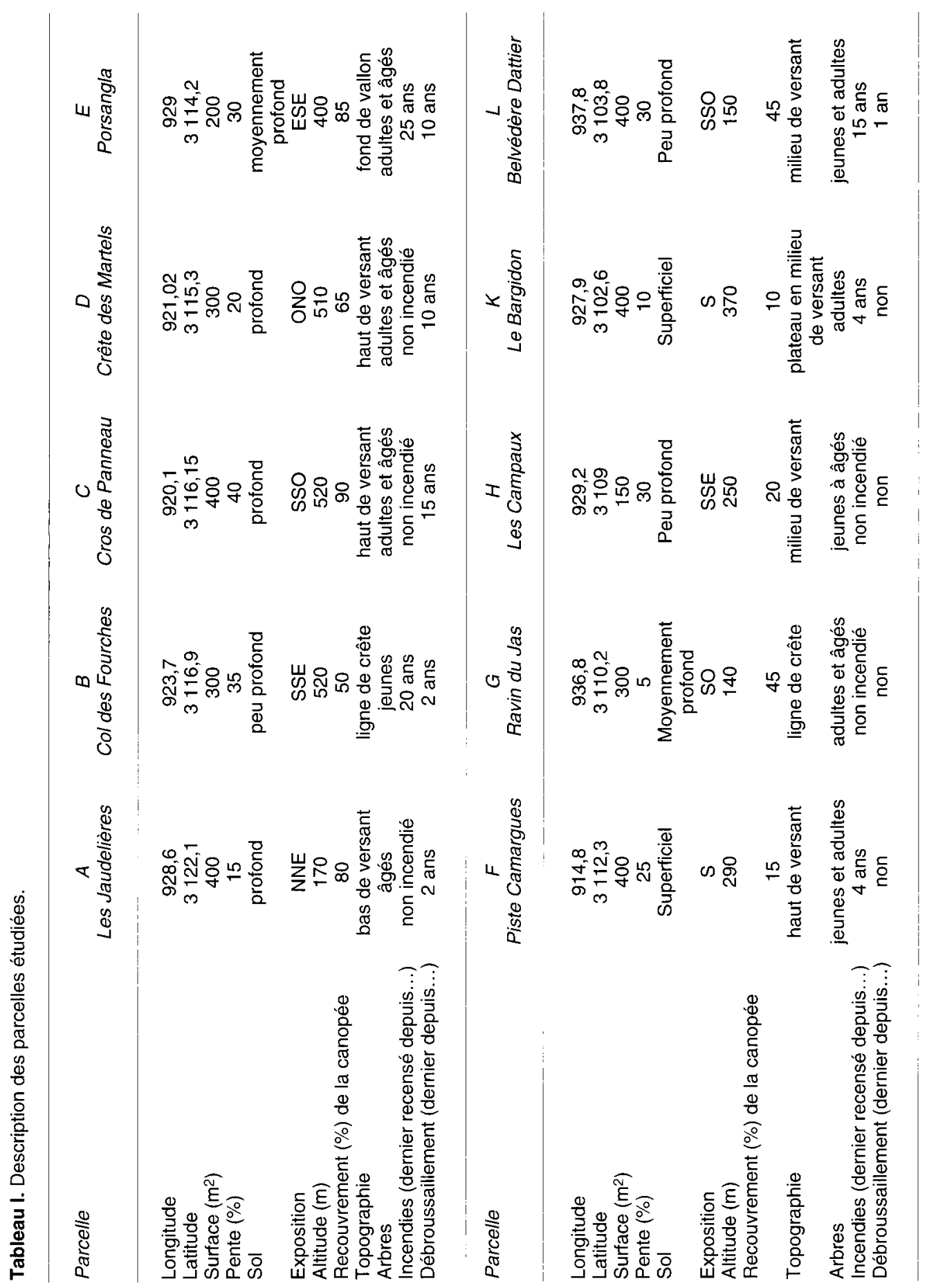


les placettes ont été regroupées en deux ensembles : placettes "humides" ou "fraîches peu dégradées» et placettes "sèches dégradées", à partir de critères basés sur leur composition floristique (les suberaies mésophiles sont marquées par la présence des Cytisus triflorus L'Héritier et $C$ monspessulanus $L$, alors que les thermophiles le sont par Genista linifolia $L$ et un cortège d'espèces des Pistacio Rhamnetalia Alaterni Riv Martinez.

Des critères dendrométriques tels que le nombre de brins, la longueur du fût, le diamètre à $1,30 \mathrm{~m}$ du sol, le diamètre du demi-fût, le recouvrement de la canopée, la hauteur de l'arbre, la longueur du levage, la circonférence au sol, la circonférence au levage, la surface terrière ont permis de choisir trois arbres représentatifs du peuplement arborescent de la placette sur lesquels les prélèvements de feuilles ont été effectués. Ceux-ci ont été réalisés aux quatre orientations principales de la canopée ainsi qu'en haut et en bas de celle-ci (soit six prélèvements de 20 feuilles par arbre).

L'ensemble des feuilles de chaque station a constitué un matériel végétal sur lequel a été effectué mensuellement l'analyse des nutriments. Les prélèvements de chaque mois et de chaque station ont été mélangés en un seul échantillon destiné aux analyses. Selon Livrelli (1993), cette stratégie donne des résultats préférables à ceux obtenus par des analyses séparées des lots de feuilles de chacun des arbres de chaque placette. Le prélèvement mensuel des feuilles a débuté au mois de novembre 1993 sur des feuilles de cette même année.

Par ailleurs, l'effet du débroussaillement a été étudié sur un ensemble de trois placettes littorales (zone géographique de la station $L$ ) à sousbois à Erica arborea L et Arbutus unedo L présentant chacune une partie débroussaillée et une autre non impactée servant de témoin. Les prélèvements ont été faits selon la même procédure que dans les autres stations mais sur six arbres au total (trois dans le témoin + trois dans la zone impactée).

Le matériel végétal récolté est constitué de feuilles entières. Celles-ci sont séchées 36 heures à $60^{\circ} \mathrm{C}$, puis broyées et soumises à une minéralisation sulfonitrique (Kjeldahl) dans le cas de $\mathrm{Ca}, \mathrm{Mg}, \mathrm{K}, \mathrm{Na}$, et à une minéralisation par $\mathrm{H}_{2} \mathrm{SO}_{4}$ dans le cas de l'azote. Par la suite, $\mathrm{Ca}, \mathrm{Mg}$, sont analysés grâce à la spectrométrie d'absorption atomique ; $\mathrm{K}$, Na par spectrométrie d'émission de flamme (en $\mathrm{mg} / \mathrm{g}$ de matière sèche). $\mathrm{N}$ est analysé grâce à l'appareil de Bouat (en $\mathrm{mg} / \mathrm{g}$ de matière sèche). La teneur en eau des feuilles est appréciée par pesée différentielle du poids frais et du poids sec, et exprimée en pourcentage.

La production de liège est estimée sur cinq des dix placettes (placettes $A, B, E, F, L$ ) par carottage sur six arbres de chacune d'elles. Afin d'avoir l'épaisseur moyenne de liège, trois carottages ont été effectués sur le tronc à 1,30 $\mathrm{m}$ du sol pour le liège primaire et le liège de reproduction (si l'arbre a été démasclé). Les stations sélectionnées s'étendent du nord au sud du massif des Maures afin d'avoir un gradient écologique.

Les données concernant les nutriments ont été traitées par des techniques d'analyse multivariées très répandues (analyse en composantes principales et analyse factorielle des correspondances) à l'aide des logiciels Statitcf et Biomeco.

\section{RÉSULTATS ET DISCUSSION}

\section{Évolution temporelle des nutriments}

La figure 1 (extraite de Livrelli 1993, d'après une étude préliminaire sur la suberaie) traduit l'évolution de la composition chimique des feuilles de $Q$ suber $L$ sur l'ensemble des stations étudiées, au cours de la première année de la feuille suivant le débourrement (mai). Les ions présents en plus grande quantité sont respectivement $\mathrm{N}, \mathrm{Ca}$,

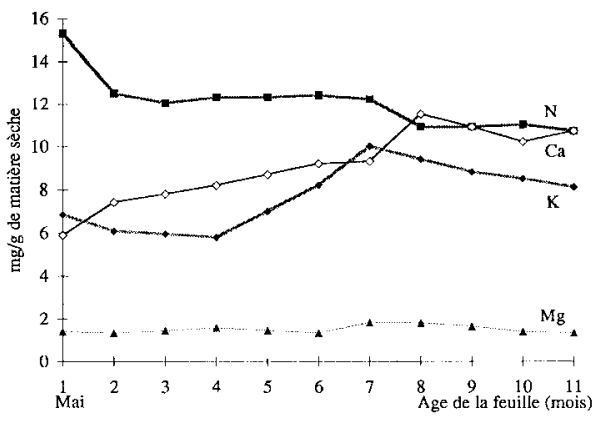

Fig 1. Composition moyenne des feuilles de $Q$ suber $L$ en nutriments. Observations faites sur une année à partir de la période de débourrement (mai) lors d'une étude préliminaire. 
et K. La concentration de l'azote est très importante au débourrement en fin de printemps (mai et juin) et décroît rapidement pour atteindre très vite un palier au cours de l'été et de l'automne, puis décroît à nouveau au cours de l'hiver. Le calcium présent en quantité non négligeable (ce qui pourrait surprendre sur substrat siliceux), s'accumule de manière continue dans les feuilles dès le débourrement. Le potassium a un comportement analogue au calcium hormis une légère regression dès l'automne. Les concentrations du magnésium restent relativement faibles et varient peu au cours du temps. Ces observations générales concordent avec les résultats observés par Martin-Prevel (1978) et Chapin (1980).

À partir des deux groupes de stations "humides peu dégradées" et "sèches dégradées" distinguées selon les critères précisés précédemment, on obtient deux séries de courbes comparées sur la figure 2 . Les stations d'ambiance «humides» (ACDEG) ou peu perturbées (il n'existe pas de forêts complètement intactes au sein du massif des Maures), les stations d'ambiance "sèche" (BFHKL) et/ou perturbées (les perturbations correspondent surtout aux incendies et aux débroussaillements) donnent des courbes d'évolution des nutriments légèrement différentes.

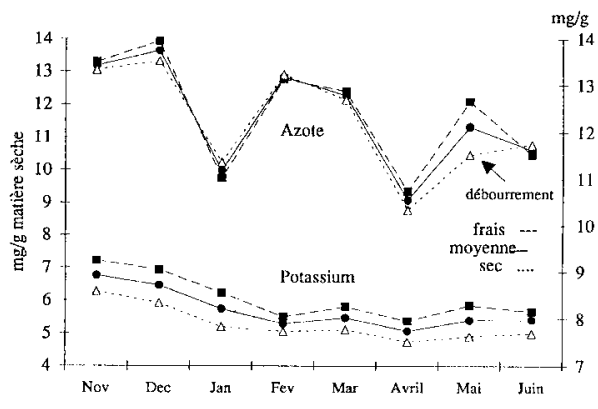

Fig 2. Évolution des teneurs en azote et en potassium des feuilles au cours du temps. Frais $=$ placettes humides peu perturbées $; \mathrm{Sec}=$ placettes sèches perturbées; Moy $=$ teneurs moyennes.
Ces variations des nutriments foliaires dans des sites dont la ou les perturbations ne sont pas provoquées à des fins expérimentales, correspondent à des perturbations inhérentes à la gestion sylvicole du massif. Les prélèvements ont débuté à l'automne sur des feuilles âgées de cinq mois, pour se poursuivre jusqu'au printemps. II s'agit donc de feuilles matures en cours d'évolution vers leur deuxième année de vie.

En ce qui concerne l'azote, la période automnale est marquée par des valeurs assez importantes (environ $13 \mathrm{mg} / \mathrm{g}$ ) expliquées par l'activité physiologique encore sensible de Quercus suber $L$ pendant cette période humide et relativement chaude (fig 2). La teneur en azote décroît ensuite jusqu'en avril pendant la période de repos hivernal, sauf pour le mois de janvier où une baisse sensible du taux d'azote marque l'effet des basses températures sur le métabolisme des arbres. Au mois de mai, on observe une nouvelle remontée des taux d'azote dans ce type de feuilles sclérophylles, traduisant la reprise du métabolisme printanier, parallèlement à l'apparition de nouvelles feuilles et de jeunes pousses, l'azote étant lié principalement à l'élaboration des protéines végétales. Les deux lots de placettes, peu dégradées humides et sèches dégradées ne sont pas significativement différentes statistiquement pour ce nutriment (tableau II).

Le potassium présente des valeurs en diminution constante au cours de la période étudiée car il est lié à la division des celIules végétales. La première année de vie des feuilles est marquée par deux phases: une augmentation de la teneur en potassium du printemps à l'automne et une diminution progressive de l'automne au printemps (fig 2). Entre novembre et juin, par trois fois, les valeurs des parcelles humides peu dégradées sont significativement supérieures aux parcelles sèches dégradées. Le potassium dans l'arbre semble donc être 
Tableau II. Test $U$ de Wilcoxon-Mann-Whitney pratiqué sur les données du groupe des placettes humides peu perturbées et sèche perturbées.

\begin{tabular}{|c|c|c|c|}
\hline Mois & $\mathrm{Ca}$ & $M g$ & K \\
\hline Nov & NS & NS & 0,075 \\
\hline Déc & 0,048 & NS & NS \\
\hline Jan & NS & NS & 0,075 \\
\hline Fév & NS & NS & NS \\
\hline Mars & NS & NS & NS \\
\hline Avril & NS & NS & NS \\
\hline Mai & NS & NS & NS \\
\hline Juin & 0,048 & NS & 0,016 \\
\hline
\end{tabular}

mieux assimilé dans les sites peu perturbés et plus humides.

Le cas du calcium est intéressant à suivre sur $Q$ suber $L$, réputé être une espèce calcifuge. On remarque au cours du vieillissement de la feuille une augmentation progressive des teneurs en calcium pendant sa première année de vie. Les teneurs restent stabilisées durant l'automne et l'hiver autour de $6 \mathrm{mg} / \mathrm{g}$, puis cet ion est accumulé dans la feuille à partir du printemps jusqu'à des teneurs voisines de $20 \mathrm{mg} / \mathrm{g}$. Sa présence dans la feuille, lors de la reprise du métabolisme de l'arbre, semble indiquer une faible capacité à stocker l'ion Ca dans les tissus ligneux, d'où sa présence excessive au niveau foliaire. En effet, le calcium (peu mobile) a un déplacement acropète dans la plante (sens ascendant), c'est pourquoi il est stocké en phase terminale au niveau de la feuille (Martin-Prevel, 1978) et au niveau des parois pecto-cellulosiques. Ce phénomène pourrait conduire, à terme, à l'intoxication des tissus foliaires en raison du caractère calcifuge de $Q$ suber $L$. De ce point de vue, il n'y a pas de différence significative entre les deux lots de placettes. L'assimilation du calcium semble indépendante des conditions stationnelles, bien que sa concentration dans les branches et les feuilles soit affectée par sa disponibilité dans le sol (De Visser, 1992).
Pour le magnésium, contrairement au calcium, les teneurs marquent une nette tendance à diminuer depuis novembre jusqu'en juin de manière régulière, sans variations marquées lors des différentes phases phénologiques des arbres et des conditions stationnelles (test non significatif du tableau II). Ce constat tend à confirmer les observations de Livrelli (1993) sur divers ligneux sclérophylles méditerranéens soulignant une régulation antagoniste du $\mathrm{Ca}$ et du Mg.

Quant au sodium, ion mineur (de 0,4 à $1,1 \mathrm{mg} / \mathrm{g}$ ) dans les végétaux de sols peu "salés", il semble être stocké plus particulièrement pendant la période de floraison de l'arbre en avril et mai (Floret et al, 1989). Les placettes humides peu dégradées ont des valeurs significativement plus grandes que les placettes sèches dégradées, uniquement pour les mois d'hiver, et ce, probablement en raison de la grande capacité du sodium à se déplacer dans les sols plus humides (voir test $U$ de Mann et Whitney, tableau III).

\section{Impact du débroussaillement}

Les nutriments principaux on été étudiés sur les parcelles choisies spécialement pour l'étude des effets du débroussaillement à 
proximité de la station $L$ (zone littorale). Cette perturbation remonte à une intervention humaine vieille d'un à trois ans maximum, après quoi le sous-bois redevient suffisamment important et la strate arbustive se referme (Gomila, 1993). L'analyse des graphiques représentant les points correspondant aux valeurs mensuelles, montre une baisse légère des valeurs de l'azote dans les feuillages des chênes de la zone impactée (TPF) pendant la période automnale et hivernale (fig 3 ). Cette baisse peut être attribuée à une diminution plus sensible, dans cette zone, de l'activité physiologique des arbres. Au printemps, la situation redevient comparable au témoin prouvant une récupération active des chênes liège de la zone débroussaillée. Contrairement à l'azote, le potassium apparaît de novembre à juin avec des valeurs supérieures à celles du feuillage du témoin (fig 3), dans la zone TPF. Cependant, les courbes ont le même tracé, mais d'une manière décalée. II en est de même pour le calcium mais de façon moins significative. Le magnésium montre des variations très différentes selon les saisons. Les teneurs sont beaucoup plus faibles dans la zone TPF que dans la zone témoin en automne et en hiver. On constate donc des variations significatives sur les bilans nutritionnels des arbres dues à l'impact de la coupe de la strate arbustive. Cette situation

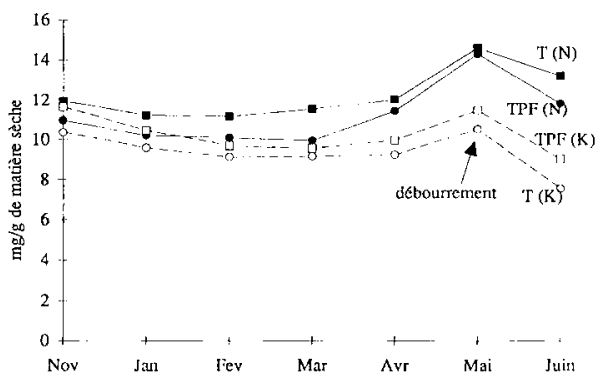

Fig 3. Évolution des teneurs en azote et en potassium des feuilles au cours du temps. TPF $=$ zone débroussaillée ; $T=$ zone témoin. des nutriments foliaires, dans la zone TPF, s'estompe progressivement dès le printemps suivant. On peut supposer que ce retour à une situation proche de celle du témoin est due à une reprise printanière de la végétation et peut-être à une réaction du métabolisme de ces végétaux

\section{Bilan hydrique foliaire}

En ce qui concerne la teneur en eau des feuilles, elle suit de manière évidente les précipitations saisonnières, ce qui paraît logique en regard de la quantité d'eau disponible dans le sol pour l'alimentation des feuilles. La séparation des parcelles en deux groupes d'ambiance différente semble être judicieuse car les parcelles humides peu dégradées ont des teneurs en eau significativement plus grandes que les parcelles sèches dégradées pour les mois d'automne et d'hiver (test significatif au seuil de risque $5 \%$ ). Au printemps, les différences «hydriques» s'estompent avec la reprise du métabolisme végétal.

\section{Synthèse des variations des nutriments}

L'analyse synthétique des résultats par l'analyse en composantes principales (ACP) (fig 4) permet de situer l'importance relative de chacune de ces variables et de comparer les parcelles en fonction de l'ensemble de ces variables. On remarque que l'axe 1 de l'analyse numérique est caractérisé par le binôme $\mathrm{Ca}-\mathrm{K}$, bien que ces deux ions aient des rôles antagonistes (l'un est antitoxique de l'autre). Ils se situent de part et d'autre du pôle positif de l'axe 1. L'axe 2, qui ne représente que $21 \%$ de l'information, est marqué négativement par le magnésium et l'eau. Dans le cas du chêne liège, il y a antagonisme entre calcium et magnésium sur le plan 1-2 de l'ACP (voir fig 4). Ce positionnement des variables peut 


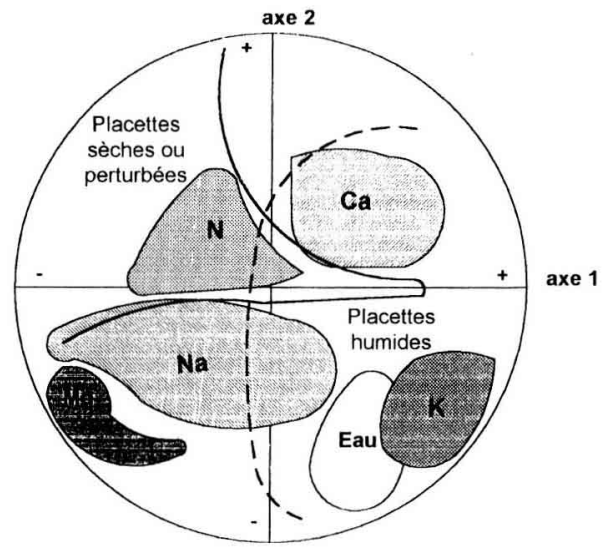

Fig 4. Plan 1-2 de l'ACP nutriments parcelles (variables nutriments représentées, interprétation synthétique du positionnement des placettes). Les placettes sèches sont sur la partie négative de l'axe 1, les placettes humides sur la partie positive de cet axe.

paraître surprenant. II confirme du point de vue fonctionnel le rôle de "maître-cation» attribué au potassium (Martin-Prevel et al, 1984), et l'importance du calcium dans le cas du chêne-liège. L'ordination des parcelles ne confirme pas nettement la discri- mination obtenue initialement à l'aide des données relatives au milieu. Il y a donc une variation, d'une parcelle à l'autre du bilan, des principaux nutriments, qui n'est pas parfaitement concordante avec la variation des paramètres écologiques (fig 4).

\section{Variation de l'épaisseur des cernes de liège}

Les figures 6 et 7 concernant l'épaisseur de liège décrivent l'évolution de la production de liège en fonction du temps dans différentes situations écologiques.

Pour le liège primaire ou mâle (fig 6), les épaisseurs cumulées les plus grandes s'observent pour des parcelles à la fois humides peu perturbées $(E, A)$ et sèches perturbées $(F)$. La production la plus faible s'observe pour une placette sèche perturbée dont les individus sont jeunes (B). La croissance du liège mâle semble donc assez peu influencée par les conditions stationnelles, mais plutôt par les variations populationnelles et de classes d'âge des arbres. Cette croissance est linéaire quelles que

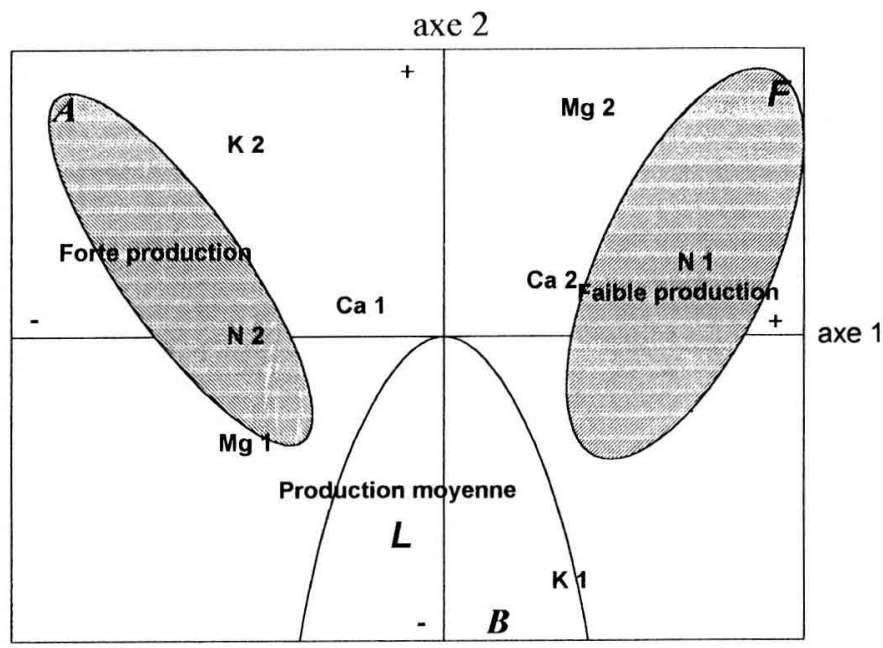

Fig 5. Plan 1-2 de l'AFC du tableau de contingence (nutriments/liège femelle). Ce plan factoriel représente en superposition les données de production (ou d'épaisseur) des cernes du liège et les teneurs en nutriments des feuilles. $1=$ classe des faibles concentrations ; 2 = classe des fortes concentrations ; $N=$ azote $\mathrm{Ca}=$ calcium ; $\mathrm{Mg}=$ magnésium $; \mathrm{K}=$ potassium. Les parcelles $A, B, L, F$ ont été positionnées en éléments supplémentaires. 
Fig 6. Courbe cumulée des épaisseurs moyennes (trois arbres) des cernes de liège mâle en fonction du temps (ans) dans les parcelles A, B, L, F, E.

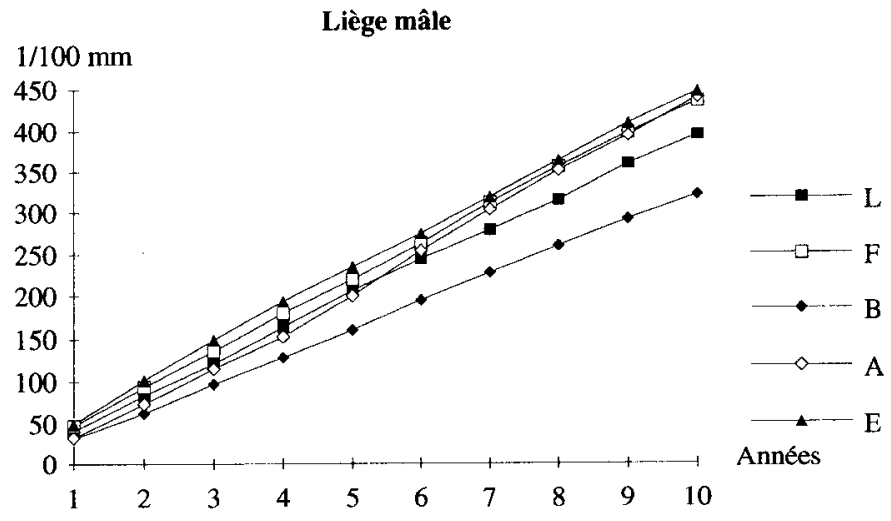

soient les conditions climatiques et les perturbations.

Pour le liège secondaire ou femelle (fig 7), au contraire, c'est la placette la plus humide non perturbée qui présente la meilleure production de liège et la placette la plus sèche perturbée qui donne les cernes les moins épais. L'épaisseur du liège femelle semble donc conditionnée à la fois par les paramètres populationnels mais aussi par les conditions climatiques de manière plus sensible que pour le liège mâle. La production de liège femelle n'est pas linéaire dans le temps (il y a augmentation de la production avec le temps et l'âge de l'arbre), mais elle reste très supérieure à la production de liège mâle.

\section{Relations croissance du liège-nutriments}

Au regard de ces hypothèses, il est intéressant d'établir des relations entre les concentrations des nutriments dans les feuilles et la croissance annuelle des cernes de liège femelle. Pour cela, il a été pratiqué une analyse factorielle des correspondances (AFC) à l'aide des tableaux de contingence établis sur les données "nutriments / liège femelle». L'AFC porte sur les parcelles A, B, $\mathrm{F}, \mathrm{L}$ pour une période de 14 ans (14 correspond au nombre minimal de cernes présents dans toutes les parcelles). Le plan 1-2 de cette AFC (fig 5) montre un gradient très net, dit «effet Guttman» (Benzecri,
Fig 7. Courbe cumulée des épaisseurs moyennes (trois arbres) des cernes de liège femelle en fonction du temps (ans) dans les parcelles $A, B, L, F$.

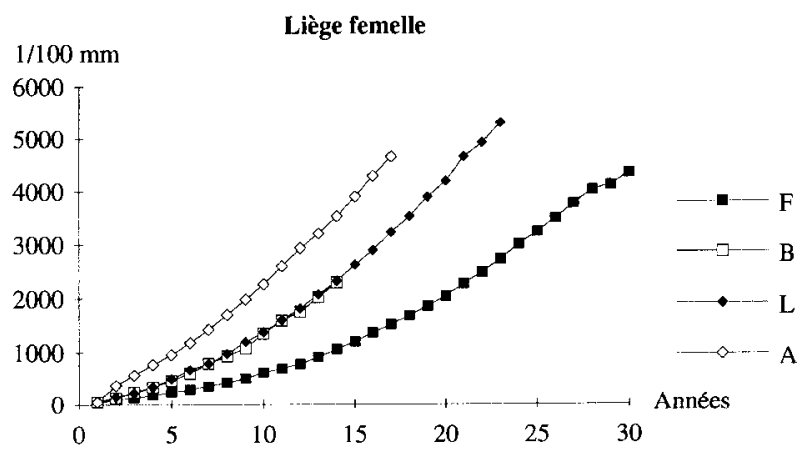


1973), entre les faibles productions dans les parcelles sèches dégradées et les fortes productions dans les parcelles humides peu dégradées. L'examen du plan 1-2 des variables nutriments par comparaison à celui des productions de liège femelle montre que les plus fortes productions de liège femelle sont liées principalement aux fortes teneurs en potassium et de manière moins prononcée aux fortes teneurs en azote du feuillage. Le magnésium semble montrer la tendance inverse. Le sodium et le calcium en revanche ne paraissent pas influencer cette production de liège.

\section{CONCLUSIONS}

Le bilan en nutriments dans les feuilles est donc vraisemblablement le plus favorable dans les parcelles humides peu perturbées, dans le massif des Maures.

Cependant, il s'agit d'une tendance générale qui doit être pondérée pour chaque ion. De plus, on constate que certaines parcelles sèches perturbées répondent favorablement au niveau des feuilles dans leur bilan nutritionnel et au niveau de la production de liège. II y a donc des exceptions liées à l'influence d'autres facteurs qui restent à définir. La production de liège femelle (qui correspond à l'exploitation de la suberaie) confirme ce bilan nuancé quant à la valeur des parcelles. La relation entre production et bilan des nutriments apparait ici évidente pour $\mathrm{K}$ et $\mathrm{N}$, mais pour les autres ions, elle devra être affinée. Les perturbations, liées à l'incendie ou au débroussaillement, ont probablement un effet négatif sur l'économie des ions dans le végétal et sur la production de liège. Ceci est à prendre en compte, dans l'avenir, pour l'aménagement des suberaies des Maures.

\section{REMERCIEMENTS}

Nous remercions l'association Forêt méditerranéenne pour son soutien financier dans ce travail.

\section{RÉFÉRENCES}

Ballini C (1993) Contribution à l'étude écologique des groupements à Ulex parviflorus Pourr en Provence calcaire : régénération, structure, productivité et dynamique des bioéléments. Thèse de doctorat, université de Provence, $257 \mathrm{p}$

Benzécri JP (1973) L'analyse des données. II. Analyse des correspondances. Dunod, Paris, $619 p$

Chapin FS (1980) The mineral nutrition of wild plants. Ann Rev Ecol Syst 11, 233-260

De Visser PHB (1992) The relations between chemical composition of oak tree rings, leaf, bark, and soil solution in a partly mixed stand. Can J For Res 22, 1824-1831

Ed-Derfoufi $F$ (1986) Gestion et dynamique des nutriments dans des taillis de chêne vert âgés et très jeunes. Thèse de $3^{e}$ cycle, université du Languedoc, $131 \mathrm{p}$

Floret $\mathrm{CH}$, Galan MJ, Le Floc'h E, Leprince F, Romane $F(1989)$ In : Plant phenomorphological studies in mediterranean type ecosystems ( $\mathrm{G}$ Orsham, ed), Kluwer Academic Publ, 9-97

Gomila H (1992) Incidences du débroussaillement sur la flore, la végétation et le sol, dans le Sud-Est de la France. Thèse de doctorat, université Aix-Marseille-III

Hasnaoui B (1992) Chênaie du nord de la Tunissie, écologie et régénération. Thèse de doctorat, université de Provence

Livrelli JN (1993) Utilisation de la méthode du diagnostic foliaire chez des végétaux ligneux sclérophylles méditerranéens pour l'étude comparée de groupements préforestiers et forestiers. Thèse de doctorat, université de Provence, $235 p$

Martin-Prevel $P$ (1978) Rôle des éléments minéraux chez les végétaux. Fruits $33,7-8,522-529$

Martin-Prevel P, Gagnard J, Gautier P (1984) L'analyse végétale dans le contrôle de l'alimentation des plantes. Tech et doc, Lavoisier

Specht RL, Rundel PW (1990) Sclerophylly and foliar nutrient status of mediterranean-climate plant communities in southern Australia. Aust J Bot 38, 459474 